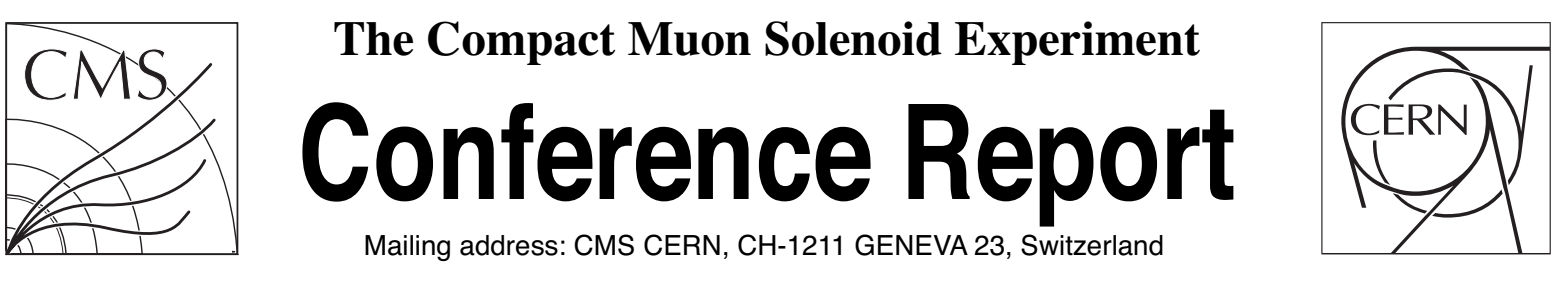

24 November 2012 (v2, 24 January 2013)

\title{
Tau trigger, reconstruction and identification at CMS
}

\author{
Cesare Calabria for the CMS Collaboration
}

\begin{abstract}
The importance of tau leptons in new physics searches at the LHC led the CMS collaboration to design a specific algorithm, the Hadron Plus Strip (HPS) algorithm, for the reconstruction and identification of taus decaying hadronically $\left(\tau_{h a d}\right)$. The HPS algorithm makes use of a particle description of the event to identify hadronic decay modes of $\tau$ leptons through the reconstruction of intermediate resonances. It also provides discriminators against potentially large backgrounds from quarks, gluons and light leptons (electrons and muons). Moreover, thanks to the particle event reconstruction avalaible in CMS, a fast online hadronic tau reconstruction can be performed allowing the development of efficient tau trigger selections which allow to perform a broad range of physics analysis. This report describes the $\tau_{\text {had }}$ reconstruction and identification of the HPS algorithm and its performances, studied in simulated $Z \rightarrow \tau \tau$ events and in samples of proton-proton collision data collected during 2011 and 2012 datataking at $\sqrt{s}=7 \mathrm{TeV}$ and $8 \mathrm{TeV}$, respectively. Finally, the performance of the tau trigger selections is presented.
\end{abstract}

Presented at TAU2012: The 12th International Workshop on Tau Lepton Physics 


\title{
Tau trigger, reconstruction and identification at CMS
}

\author{
Cesare Calabria $^{\mathrm{a}}$, on behalf of the CMS Collaboration
}

${ }^{a}$ INFN and University of Bari, Italy

\begin{abstract}
The importance of tau leptons in new physics searches at the LHC led the CMS collaboration to design a specific algorithm, the Hadron Plus Strip (HPS) algorithm, for the reconstruction and identification of taus decaying hadronically $\left(\tau_{\text {had }}\right)$. The HPS algorithm makes use of a particle description of the event to identify hadronic decay modes of $\tau$ leptons through the reconstruction of intermediate resonances. It also provides discriminators against potentially large backgrounds from quarks, gluons and light leptons (electrons and muons). Moreover, thanks to the particle event reconstruction avalaible in CMS, a fast online hadronic tau reconstruction can be performed allowing the development of efficient tau trigger selections which allow to perform a broad range of physics analysis. This report describes the $\tau_{\text {had }}$ reconstruction and identification of the HPS algorithm and its performances, studied in simulated $Z \rightarrow \tau \tau$ events and in samples of proton-proton collision data collected during 2011 and 2012 data-taking at $\sqrt{s}=7 \mathrm{TeV}$ and $8 \mathrm{TeV}$, respectively. Finally, the performance of the tau trigger selections is presented.
\end{abstract}

Keywords: CMS, LHC, Tau, Physics, Software, Computing

\section{Introduction}

The primary goal of the Compact Muon Solenoid (CMS) [1] experiment is to explore particle physics at the $\mathrm{TeV}$ energy scale by studying the final states produced in the proton-proton collisions at the Large Hadron Collider (LHC) [2]. Tau leptons provide an experimentally favourable signature for these studies. Moreover, because of their higher mass than other leptons, taus play a crucial role in the searches for the Standard Model (SM) Higgs boson, especially in the low mass region $\left(M_{H}<130 \mathrm{GeV} / \mathrm{c}^{2}\right)$, where $H \rightarrow \tau \tau$ decay mode has the second highest branching ratio, after the decay in $b \bar{b}$ which is more challenging to reconstruct. In the recent discovery of a new boson with mass $125.3 \pm 0.6 \mathrm{GeV} / \mathrm{c}^{2}$ compatible with the Standard Model (SM) Higgs boson at CMS [3] and ATLAS [4], coupling to fermions is still not confirmed. Therefore the nature of this new particle can be clarified by $H \rightarrow \tau \tau / b \bar{b}$ channels. $H \rightarrow \tau \tau$ is the most sentive channel among all the fermionic channels, confirming the need for a efficient and accurate reconstruction and identification of taus.
The lifetime of $\tau$ leptons is short enough that they decay before reaching the detector elements. In two thirds of the cases $\tau$ leptons decay hadronically, typically into one or three charged mesons (predominantly $\pi^{+}, \pi^{-}$), often accompanied by neutral pions (decaying via $\pi^{0} \rightarrow \gamma \gamma$ ) and a $v_{\tau}$ (that escapes the reconstruction), while the other cases involve muons and electrons. Hadronic decays of the tau lepton are usually via the $\rho(770)$ or $a_{1}(1200)$ resonances. The tau decay modes and the relative branching ratios are presented in Table 1 . Taus produced from heavy particle decays such as the $Z$ or Higgs bosons are boosted, therefore they appear in the detector as a highly collimated jets, also characterized by high isolation and low charged track multiplicity.

\section{Particle flow}

The particle flow event reconstruction aims at reconstructing and identifying all stable particles in the event that are classified into mutually exclusive categories 
Table 1: BR of the dominant decays of the $\tau$ lepton. The $h$ stands for both $\pi$ and Kaon. The table is symmetric under charge conjugation[5].

\begin{tabular}{l|c|c|c}
\hline \hline Decay mode & Resonance & Mass $\left(\mathrm{MeV} / \mathrm{c}^{2}\right)$ & BR (\%) \\
\hline$\tau^{-} \rightarrow e^{-} \bar{v}_{e} v_{\tau}$ & & & $(17.83 \pm 0.04) \%$ \\
$\tau^{-} \rightarrow \mu^{-} \bar{v}_{\mu} v_{\tau}$ & & & $(17.41 \pm 0.04) \%$ \\
\hline$\tau^{-} \rightarrow h^{-} v_{\tau}$ & & & $(11.53 \pm 0.06) \%$ \\
$\tau^{-} \rightarrow h^{-} \pi^{0} v_{\tau}$ & $\rho^{-}$ & 770 & $(25.95 \pm 0.09) \%$ \\
$\tau^{-} \rightarrow h^{-} \pi^{0} \pi^{0} v_{\tau}$ & $a_{1}^{-}$ & 1200 & $(9.52 \pm 0.11) \%$ \\
$\tau^{-} \rightarrow h^{-} h^{+} h^{-} v_{\tau}$ & $a_{1}^{-}$ & 1200 & $(9.80 \pm 0.07) \%$ \\
$\tau^{-} \rightarrow h^{-} h^{+} h^{-} \pi^{0} v_{\tau}$ & & & $(4.76 \pm 0.06) \%$ \\
\hline \hline
\end{tabular}

(electrons, muons, photons, charged hadrons and neutral hadrons) with a thorough combination of all CMS sub-detectors towards an optimal determination of their direction, energy and type. This list of individual particles is then used to build jets, to determine the missing transverse energy $E_{T}^{\text {miss }}$, which gives an estimate of the direction and energy of the neutrinos and other invisible particles, to reconstruct and identify taus from their decay products, to quantify charged lepton isolation with respect to other particles, to tag jets coming from the $b$ quark decay. Particle flow algorithm is able to tag charged particles from pile-up (PU), minimizing the impact of PU on jet reconstruction and lepton or photon isolation [6].

\section{The HPS algorithm}

The HPS algorithm [7] starts the decay mode reconstruction from the constituents of a PF jet and it gives special attention to photon conversions in the CMS tracker material. The bending of electron/positron tracks in the magnetic field of the CMS solenoid broadens the calorimeter signatures of neutral pions in the azimuthal direction. This effect is taken into account in the HPS algorithm by reconstructing photons in "strips", objects that are built out by organizing electromagnetic particles (PF photons and electrons) inside the PF jet within a window of size $\Delta \eta=0.05 \times \Delta \phi=0.20$. Strips satisfying a minimum transverse momentum are finally combined with the charged hadrons to reconstruct individual $\tau_{\text {had }}$ the following decay modes:

1. Single hadron corresponds to $h^{-} v_{\tau}$ and $h^{-} \pi^{0} v_{\tau}$ decays where $\pi^{0}$ has too little energy to be reconstructed as strips.

2. One hadron + one or two strips reconstructs the decay mode $h^{-} \pi^{0} v_{\tau}$, depending if the photons from $\pi^{0}$ decay are well separated on the calorimeter surface.

3. Three hadrons corresponds to the decay mode $h^{-} h^{+} h^{-} v_{\tau}$. The three charged hadrons are required to come from the same secondary vertex.
Other decay modes, $h^{-} \pi^{0} \pi^{0} v_{\tau}$ and $h^{-} h^{+} h^{-} \pi^{0} v_{\tau}$, are reconstructed via the previous topologies. All charged hadrons and strips are required to be contained within a cone of size that depends on the transverse momentum of the reconstructed $\tau_{\text {had }}$. The reconstructed tau momentum is required to match the $(\eta, \phi)$ direction of the original PF jet. Moreover charged hadrons are supposed to be pions, strips must be compatible with the $\pi^{0}$ mass and the hadron+strip system is required to be consistent with the mass of one of the intermediate meson resonances listed in Table 1.

To discriminate genuine $\tau_{\text {had }}$-leptons from quark and gluon jets in QCD multijet events, the $\tau_{\text {had }}$ candidate is required to be isolated. Two types of isolation are used in HPS: a cut-based and a so-called "ring-based" isolation. The cut-based approach consists on selections on the sum $p_{T}$ of charged, neutral and gamma PF candidates not belonging to tau within a cone around the reconstrcuted $\tau_{\text {had }}$. The energy contribution from neutral particles is corrected by subtracting the energy of charged candidates coming from pile-up vertices in a cone around the tau candidate, multiplied by a neutralto-charged ratio tuned to obtain flat efficiency vs. pileup ( $\Delta \beta$ correction).

The ring-based isolation consists of a multivariate Boosted Decision Tree (BDT) [8] discriminator based on the energy deposits of the reconstructed PF candidates in rings around the identified $\tau_{\text {had }}$ candidate, mean and variance of their position in the $\eta-\phi$ plane and the energy density $\rho$ in the event to reduce the dependency of the isolation efficiency on pile-up [9].

The constituents of the $\tau_{\text {had }}$ are always excluded from the calculation of the isolation variables. Different working points (WP) are defined for both the isolations (loose, medium, tight), tuned in order to give different levels of fake-rate from jets.

Electrons and muons can be misidentified as pions and reconstructed as $\tau_{\text {had }}$ in the HPS algorithm. To distinguish genuine $\tau_{\text {had }}$-leptons from light leptons additional discriminator working points have been defined and implemented.

For the discrimination against muons three working points are defined in order to avoid the leading track of the reconstructed $\tau_{\text {had }}$ is also reconstructed in the CMS muon system. In the case of single hadron decay mode, the tighter discriminator includes a veto against minimum ionizing particles (MIP) based on the ratio of the calorimeter energy deposits over the track momentum of the leading charged PF candidate.

The discrimination against electrons is performed through a cut-based approach or a dedicated multivariate discriminator. In the cut-based approach three dif- 
ferent WPs are defined that takes advantage of an electron/pion multivariate discriminant $(\xi)$ implemented in the PF algorithm that exploits calorimetric and tracking informations. The tighter WP includes also specific cuts to reduce charged hadrons faking the single hadron decay mode and high brem electrons faking single hadron plus strip decay mode.

The multivariate discriminator is based on the output of a multivariate BDT. First the $\tau_{\text {had }}$ candidates are categorized on the basis of the number of neutral PF constituents, the pseudorapidity and the presence of an electron reconstructed in the tracker associated to the leading charged hadron candidate. Depending on the object category, the BDT is trained on a dedicated set of variables that include also the $\xi$ discriminator and other calorimetric and tracking informations.

The efficiency of the HPS working points has been evaluated with genuine $\tau_{\text {had }}$ candidates taken from simulated $Z \rightarrow \tau \tau$ events and the results of this measurement is depicted in Fig. 1. The ring-based isolation has been trained against jet $\rightarrow \tau_{\text {had }}$ fakes with similar $\tau_{\text {had }}$ candidates and with multijet events in data. The fakerate level from jet achievable with the MVA isolation is expected to be $25-30 \%$ lower than the one obtained using the cut based isolation. Moreover, once the jet $\rightarrow$ $\tau_{\text {had }}$ fake-rate level is fixed, the MVA approach provides the highest identification efficiency $(\sim 70 \%)$. Tau identification efficiency has been measured also in data with a "tag-and-probe" technique [10] in $Z \rightarrow \tau \tau \rightarrow \mu \tau_{\text {had }}$ events. Good agreement between data and simulation is found and the relative systematic uncertainty is $\sim 6 \%$.

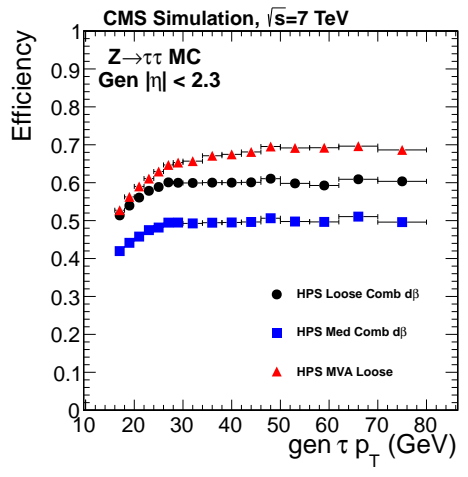

Figure 1: Expected tau efficiency from $Z \rightarrow \tau \tau$ MC for different WPs.

Robustness of the HPS reconstruction and identification against pile-up has been also tested in $Z \rightarrow \tau \tau$ events. As shown in Fig. 2 top, the identification efficiency has very weak dependence on pile-up. Thanks to the high precision reconstruction provided by the $\mathrm{PF}$ algorithm, also the reconstructed $\tau_{\text {had }}$ energy is minimally affected by pile-up effects, see Fig. 2 bottom.
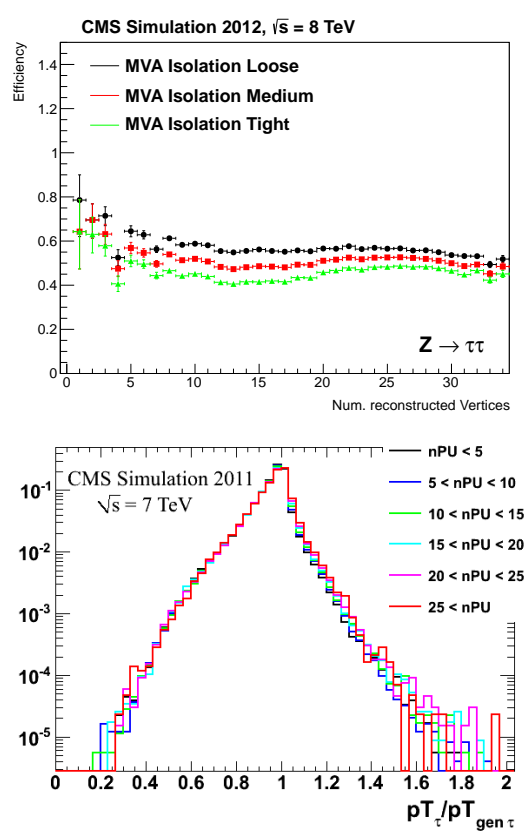

Figure 2: Tau ID efficiency vs. number of reconstructed primary vertices with the MVA approach (top) and $p_{T}$ resolution for 1-prong +1 $\pi^{0}$ taus in $Z \rightarrow \tau \tau$ MC events for different PU scenarios (bottom).

Jet $\rightarrow \tau_{\text {had }}$ fake-rate has been measured in data in events with one isolated muon ( $W \rightarrow \mu v+$ jets sample). The results are shown in Fig. 3 demonstrating that jet fake-rate is lower than $3 \%$ and for the MVA isolation is $10-20 \%$ lower than cut-based isolation.

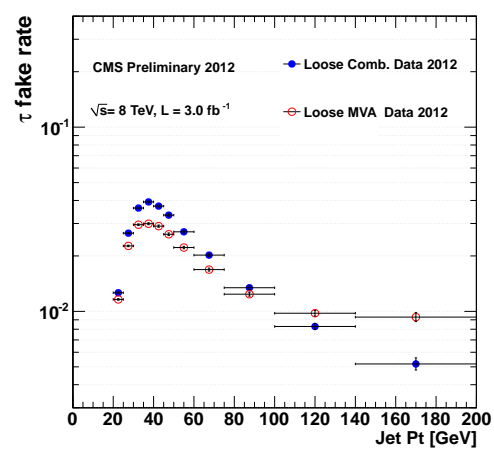

Figure 3: Jet $\rightarrow \tau_{\text {had }}$ fake-rate measured in $W \rightarrow \mu v+$ jets events parameterized in terms of the associated PF jet $p_{T}$.

The fake-rate efficiency from electrons (muons) has been measured in data with a tag-and-probe technique using a sample of isolated electrons (muons) coming from $Z \rightarrow e e(\mu \mu)$ decay. The $e \rightarrow \tau_{\text {had }}$ fake-rate is 
found to be lower in the CMS barrel region than in the endcap regions and ranges from $\sim 13 \%(\sim 29 \%)$ for the loose anti-electron discriminator to $\sim 1 \%(\sim 2 \%)$ for the MVA anti-electron discriminator in the barrel (endcap) region. The $\mu \rightarrow \tau_{\text {had }}$ fake-rate efficiency is very low and difficult to measure, especially for the tighter WPs, and it has been found to be $\sim 2$ per mille.

\section{Tau triggers}

The CMS Collaboration developed efficient HLT [11] trigger selections involving one or two hadronic taus in coincidence with other physics objects. In particular, the online coincidence of a lepton (muon or electron) and a hadronic $\left(l+\tau_{\text {had }}\right.$ cross-triggers $)$ tau spatially separated and satisfying specific quality and kinematical cuts allows for a efficient rate reduction, keeping the kinematic thresholds for both objects small, as required by the offline analyses. Hadronic taus employed in the tau triggers are reconstructed with the PF algorithm [12] which is fast enough to be used at HLT level.

The efficiency of $\mu+\tau_{\text {had }}$ and $e+\tau_{\text {had }}$ cross-triggers has been measured with a tag-and-probe technique and $Z \rightarrow \tau \tau \rightarrow \mu / e+\tau_{\text {had }}$ in two different periods of the 2012 data-taking characterized by different instantaneous luminosity (Run2012A with $5 \times 10^{33} \mathrm{~cm}^{-2} \mathrm{~s}^{-1}$ and Run2012B with $7 \times 10^{33} \mathrm{~cm}^{-2} \mathrm{~s}^{-1}$ ). The two $l+\tau_{\text {had }}$ trigger paths are higly efficient, thanks to the PF algorithm, with a narrow turn-on, see Fig. 4.

\section{Conclusions}

The CMS collaboration has developed an advanced and robust reconstruction and identification algorithm for hadronic taus, which has been successfully commissioned with the data collected in 2011 and 2012. Different isolation approaches are avalaible, the wellestablished cut-based isolation or the MVA ring-based isolation, which provides an identification efficiency up to $70 \%$ with a fake-rate from jets lower than $3 \%$. Antimuon and anti-electron discriminators are provided as well with several working points suitable for the different background levels. Moreover, very efficient tau triggers are avalaible for analyses involving hadronic taus.

\section{References}

[1] R. A. et al., The CMS experiment at the CERN LHC, JINST 03 (2008) S08004. doi:10.1088/1748-0221/3/08/S08004.

[2] L. Evans, P. Bryant, LHC Machine, JINST 03 (2008) S08001. doi:10.1088/1748-0221/3/08/S08001.
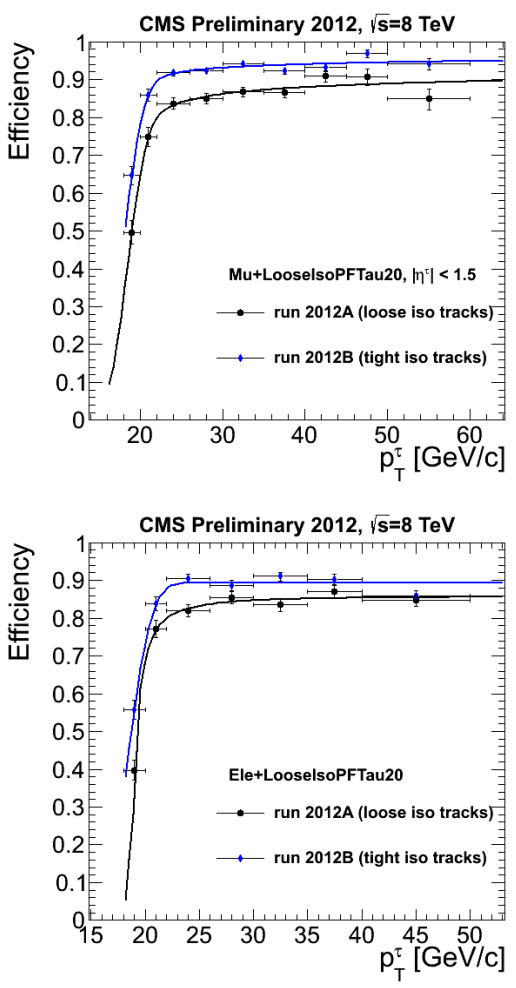

Figure 4: $\mu+\tau_{\text {had }}$ turn-on curve in the barrel barrel region (top) and $e+\tau_{\text {had }}$ turn-on curve in the barrel plus endcap region (bottom).

[3] S. Chatrchyan, et al., Observation of a new boson at a mass of $125 \mathrm{GeV}$ with the CMS experiment at the LHC, Phys.Lett. B716 (2012) 30-61. arXiv:1207.7235, doi:10.1016/j.physletb.2012.08.021.

[4] G. Aad, et al., Observation of a new particle in the search for the Standard Model Higgs boson with the ATLAS detector at the LHC, Phys.Lett. B716 (2012) 1-29. arXiv:1207.7214, doi:10.1016/j.physletb.2012.08.020.

[5] J. B. et al., (PDG), PR D86, 010001 (2012).

[6] CMS Collaboration, Particle-Flow Event Reconstruction in CMS and Performance for Jets, Taus, and MET.

[7] CMS Collaboration, Performance of $\tau$-lepton reconstruction and identification in CMS, Journal of Instrumentation 7 (2012) 1001. arXiv:1109.6034, doi:10.1088/1748-0221/7/01/P01001.

[8] B. P. e. a. Roe, Boosted Decision Trees as an Alternative to Artificial Neural Networks for Particle Identification, Nucl. Instrum. Meth A543 (2005) 577. arXiv:physics/0408124v2, doi:doi:10.1016/j.nima.2004.12.018.

[9] M. Cacciari, G. P. Salam, Pileup subtraction using jet areas, Phys.Lett. B659 (2008) 119-126. arXiv:0707.1378, doi:10.1016/j.physletb.2007.09.077.

[10] J. e. a. Berryhill, Generic Tag and Probe Tool for Measuring Efficiency at CMS with Early Data, CMS Note 2009/111.

[11] CMS Collaboration, CMS High Level Trigger, LHCC Report CERN-LHCC-2007-021 (2007).

[12] CMS Collaboration, CMS Strategies for tau reconstruction and identification using particle-flow techniques. 